\title{
Morphometry of the Anterior Third Ventricle Region as a Guide for the Transcallosal-Interforniceal Approach
}

\author{
Mete ERTurK, Gulgun KAyAlioglu, Mehmet Asim OzER, and Tomris OzGuR
}

Department of Anatomy, Ege University Faculty of Medicine, Bornova, Izmir, Turkey

\begin{abstract}
The transcallosal-interforniceal approach is the most appropriate approach to localize and totally remove space-occupying lesions around the anterior third ventricle region such as craniopharyngiomas and gliomas. This study examined the microsurgical anatomy of the normal anterior third ventricle region in 81 adult cadaver hemispheres. The central sulcus was identified and surface landmarks determined as the points $5 \mathrm{~cm}(\mathrm{P5})$ and $7 \mathrm{~cm}$ anterior to the central sulcus (P7). The distances between $\mathrm{P5}$ and $\mathrm{P7}$ and the upper margin of the interventricular foramen, which delineate the surgical corridor chosen to avoid disturbance of important neural structures, were 46.26-60.96 (54.09 \pm 3.35$) \mathrm{mm}$ and 48.00-62.00 $(54.94 \pm 3.09) \mathrm{mm}$, respectively. The distances between the upper margin of the hemisphere and the cingulate sulcus, especially important for avoiding damage to the cingulate gyrus and other mesiolimbic structures, were 13.54-30.00 (21.28 \pm 3.89) $\mathrm{mm}$ and 12.22-29.52 (21.12 \pm 3.90) $\mathrm{mm}$ at the level of P5 and P7. The distances between the upper margin of the hemisphere and the callosal cistern containing the pericallosal artery were $28.34-40.50(33.94 \pm 2.84) \mathrm{mm}$ and $28.16-40.26$ $(33.50 \pm 2.61) \mathrm{mm}$, respectively. Normative morphometric data of the structures involved in the surgical procedure are necessary for planning and performance of the transcallosal-interforniceal approaches. This study of a large series of specimens shows that these measurements have large individual variations.
\end{abstract}

Key words: anatomy, morphometry, third ventricle, transcallosal-interforniceal approach

\section{Introduction}

Various benign and malignant tumors and cyst formations affect the anterior third ventricle region. The most common primary tumors of the region are craniopharyngiomas and gliomas, followed by ependymomas, colloid cysts, and choroid plexus papillomas. Secondary tumors in and around the anterior part of the third ventricle expanding within the ventricle include meningiomas, optic nerve and hypothalamic gliomas, pituitary adenomas, and craniopharyngiomas. ${ }^{6}$

Benign and malignant neoplasms of the third ventricle can be removed with well-planned microsurgical approaches. There are various operative approaches to the anterior part of the third ventricle as follows: Transcortical-transventricular approach through the interventricular foramen, transcallosal-transforaminal approach, transcallosal-interforniceal approach, transcallosal-subchoroidal transvelum interpositum approach, subfrontal trans-lamina terminalis approach, pterional

Received July 30, 2003; Accepted January 26, 2004 approach, and combinations of these approaches. In the last decade, the transcortical-transventricular, transcallosal, and transcallosal-interforniceal approaches have been preferred. The transcallosalinterforniceal approach gives excellent results and is accepted as the most appropriate approach to localize and totally remove space-occupying lesions of the anterior third ventricle. ${ }^{1,5,10,11)}$

The third ventricle is one of the most surgically inaccessible areas in the brain, and is impossible to reach without incising some neural structures. A good understanding of the anatomy of the transcallosal-interforniceal approach is important to preserve important anatomic structures such as the pyramidal tract, genu of the corpus callosum, commissure and columns of the fornix, and the anterior commissure. Normative data of this region is necessary to evaluate changes in the anatomy due to the pathological process because large lesions can distort the topography and landmarks. However, there are very few studies on the morphometry of the region. ${ }^{11,12)}$

This study examined the microsurgical anatomy of the normal anterior third ventricle region that is 


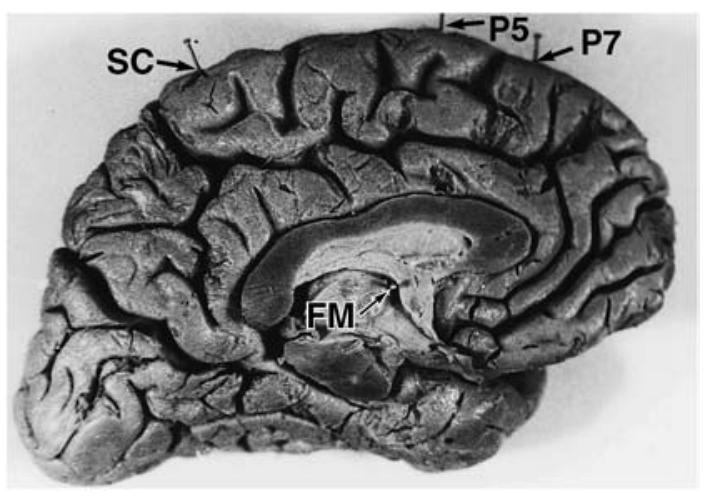

Fig. 1 Reference points for measurements in the transcallosal-interforniceal approach. FM: interventricular foramen, P5: point 5 cm anterior to the central sulcus, P7: point 7 cm anterior to the central sulcus, SC: central sulcus.

surgically important in the transcallosal-interforniceal approach in a large series of cadaver specimens.

\section{Materials and Methods}

This study used 81 adult cadaver cerebral hemispheres without macroscopic pathology, fixed with $10 \%$ formalin for several months, from the collection of the Department of Anatomy, Ege University Faculty of Medicine. The central sulcus was identified, and surface landmarks were determined as the points $5 \mathrm{~cm}$ anterior to the central sulcus (P5) and $7 \mathrm{~cm}$ anterior to the central sulcus (P7) and marked with needles, in accordance with the previous study ${ }^{11)}$ (Fig. 1). Morphometric measurements were made for structures on the lines joining the P5 and the upper margin of the interventricular foramen (FM) and the P7 and FM, which represent the path of the transcallosal-interforniceal approach chosen to avoid disturbance of important neural structures, such as the genu of the corpus callosum, the commissure of the fornix, and the motor strip.

The points where the P5-FM and P7-FM lines intersected with the corpus callosum were labeled A and $\mathrm{B}$ (Fig. 2). ${ }^{11)}$ Other important anatomical landmarks are explained in Fig. 2 and Tables 1 and 2. Various measurements were made between these landmarks to characterize the anatomy of this region.

All measurements were taken using a ruler under a Jena 390822 X 6.3 (Jena, Germany) dissecting microscope. Mean and standard deviation values, and maximum and minimum values were calculated

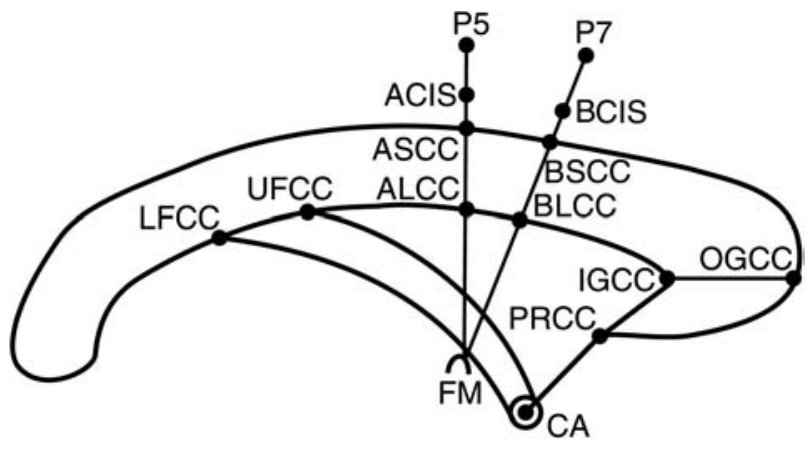

Fig. 2 Schematic illustration of the measurements in the transcallosal-interforniceal approach. ACIS: intersection point between the P5-FM line and the cingulate sulcus, ALCC: intersection point between the P5FM line and the lower surface of the corpus callosum, ASCC: intersection point of the P5-FM line with the upper surface of the corpus callosum, BCIS: intersection point between the P7-FM line and the cingulate sulcus, BLCC: intersection point of P7-FM line with the lower surface of the corpus callosum, BSCC: intersection point between the P7-FM line and the upper surface of the corpus callosum, CA: anterior commissure, FM: interventricular foramen, IGCC: most anterior point of the inner surface of the genu of the corpus callosum, LFCC: lower insertion point of the fornix to the corpus callosum, OGCC: most anterior point of the outer surface of the genu of the corpus callosum, P5: point $5 \mathrm{~cm}$ anterior to the central sulcus, P7: point $7 \mathrm{~cm}$ anterior to the central sulcus, PRCC: most posterior point of the rostrum of the corpus callosum, UFCC: upper insertion point of the fornix to the corpus callosum.

for each parameter.

\section{Results}

The distances between brain structures on the P5FM and P7-FM lines delineating the transcallosalinterforniceal surgical corridor and surrounding structures are shown in Table 1. Distances to brain structures around the A and B points where the P5FM and P7-FM lines intersect with the lower border of the corpus callosum are shown in Table 2 .

The P5-ACIS and P7-BCIS distances between the upper margin of the hemisphere and the cingulate sulcus are especially important for avoiding damage to the cingulate gyrus and other mesiolimbic structures. P5-ASCC and P7-BSCC are the distances 
Table 1 Measurements along the P5-FM and P7-FM lines in the present and previous studies

\begin{tabular}{lccc}
\hline & & Distance $(\mathrm{mm})$ & \\
\cline { 2 - 4 } Landmarks & Present study & Winkler et al. (1997) & Winkler et al. (1999) \\
\hline P5-FM & $54.09 \pm 3.35(46.26-60.96)$ & not measured & $62.99 \pm 4.52(51.28-76.72)$ \\
P7-FM & $54.94 \pm 3.09(48-62)$ & not measured & $63.84 \pm 4.15(55.26-74.8)$ \\
P5-ACIS & $21.28 \pm 3.89(13.54-30)$ & $23.96 \pm 4.06(15-32)$ & $25.76 \pm 4.82(17.13-42.73)$ \\
P7-BCIS & $21.12 \pm 3.9(12.22-29.52)$ & $24.29 \pm 3.92(20-35)$ & $25.41 \pm 4.94(12.91-36.29)$ \\
P5-ASCC & $33.94 \pm 2.84(28.34-40.50)$ & $37.71 \pm 3.29(31-44)$ & $38.67 \pm 3.57(31.76-50.14)$ \\
P7-BSCC & $33.50 \pm 2.61(28.16-40.26)$ & $37.52 \pm 3.10(32-45)$ & $38.33 \pm 3.96(29.17-50.26)$ \\
ACIS-ASCC & $12.51 \pm 3.01(6.54-19.22)$ & $13.50 \pm 3.51(8-20)$ & $12.91 \pm 3.64(7.19-22.6)$ \\
BCIS-BSCC & $12.13 \pm 3.48(5.90-20.60)$ & $12.73 \pm 3.17(6-18)$ & $12.92 \pm 3.67(6.75-23.37)$ \\
ASCC-ALCC & $6.29 \pm 1.13(4.20-9.76)$ & $6.12 \pm 1.19(4-8)$ & $6.22 \pm 1.27(3.07-9.0)$ \\
\hline
\end{tabular}

Values are mean \pm standard deviation (range). ACIS: intersection point between the P5-FM line and the cingulate sulcus, ALCC: intersection point between the P5-FM line and the lower surface of the corpus callosum, ASCC: intersection point of the P5-FM line with the upper surface of the corpus callosum, BCIS: intersection point between the P7-FM line and the cingulate sulcus, BSCC: intersection point between the P7-FM line and the upper surface of the corpus callosum, FM: interventricular foramen, P5: point $5 \mathrm{~cm}$ anterior to the central sulcus, P7: point $7 \mathrm{~cm}$ anterior to the central sulcus.

Table 2 Measurements around points $A$ and $B$ at the intersections of the P5-FM and P7-FM lines with the lower border of the corpus callosum in the present and previous studies

\begin{tabular}{lccc}
\hline & & Distance $(\mathrm{mm})$ & \\
\cline { 2 - 4 } Landmarks & Present study & Winkler et al. (1997) & Winkler et al. (1999) \\
\hline ALCC-UFCC & $19.84 \pm 8.11(6.4-39.36)$ & $23.79 \pm 8.09(10-40)$ & $29.62 \pm 9.79(9.98-47.72)$ \\
ALCC-LFCC & $28.84 \pm 5.61(16.46-40.90)$ & not measured & not measured \\
BLCC-UFCC & $25.57 \pm 8.18(10-46)$ & $28.83 \pm 8.18(16-50)$ & $34.71 \pm 10.15(13.82-54.52)$ \\
BLCC-LFCC & $34.63 \pm 5.60(23.04-46.20)$ & not measured & not measured \\
ALCC-CA & $16.85 \pm 3.22(11.06-28.46)$ & $16.04 \pm 3.00(12-25)$ & $23.46 \pm 3.74(11.98-32.7)$ \\
BLCC-CA & $16.30 \pm 3.04(11.22-27.36)$ & $16.79 \pm 3.13(13-29)$ & $22.89 \pm 3.74(11.05-33.04)$ \\
CA-FM & $3.36 \pm 0.62(1.9-5.54)$ & $4.96 \pm 1.88(2.5-10)$ & $6.78 \pm 2.64(1.86-14.57)$ \\
PRCC-CA & $8.21 \pm 1.53(5-11.22)$ & not measured & $13.37 \pm 2.89(3.87-18.66)$ \\
IGCC-OGCC & $11.20 \pm 1.52(7.40-15.20)$ & not measured & $12.61 \pm 3.1(6.42-23.15)$ \\
CL & $68.42 \pm 5.17(45.32-79.72)$ & not measured & $74.93 \pm 5.49(61.86-86.06)$ \\
\hline
\end{tabular}

Values are mean \pm standard deviation (range). ALCC: intersection point between the P5-FM line and the lower surface of the corpus callosum, BLCC: intersection point of P7-FM line with the lower surface of the corpus callosum, CA: anterior commissure, CL: length of the corpus callosum, FM: interventricular foramen, IGCC: most anterior point of the inner surface of the genu of the corpus callosum, LFCC: lower insertion point of the fornix to the corpus callosum, OGCC: most anterior point of the outer surface of the genu of the corpus callosum, P5: point $5 \mathrm{~cm}$ anterior to the central sulcus, P7: point $7 \mathrm{~cm}$ anterior to the central sulcus, PRCC: most posterior point of the rostrum of the corpus callosum, UFCC: upper insertion point of the fornix to the corpus callosum.

between the upper margin of the hemisphere and the callosal cistern, which contain the pericallosal artery.

\section{Discussion}

The results of our study of the structures along the P5-FM and P7-FM lines are compared with the previous studies of 30 formalin-fixed adult human brains and on 72 adult mid-sagittal magnetic resonance images (Tables 1 and 2). ${ }^{11,12)}$ Another study measured the distance between the interventricular foramen and the anterior commissure as $3.49 \mathrm{~mm}$ (range 2.0-5.0 mm). ${ }^{7)}$ As shown, the measurements of the present study are close to a 1997 study performed on 30 formalin-fixed cadavers, ${ }^{11)}$ but smaller compared to the later 1999 study performed on 72 mid-sagittal magnetic resonance 
specimens $^{12)}$ (Tables 1 and 2). Distances ALCC-CA (distance of the intersection point between the P5FM line and the lower surface of the corpus callosum to the anterior commissure) and BLCC-CA (distance of the intersection point between the P7FM line and the lower surface of the corpus callosum to the anterior commissure) are significantly higher in the 1999 study $^{12)}$ when compared to both our study and the 1997 study. ${ }^{11)}$ The measurements probably differ due to differences in the populations investigated. Cadaver specimens provide valuable information despite the differences from patients undergoing surgery due to the absence of blood flow, postmortem changes in the vascular and neural structures, and changes due to formalin fixation. A study of the ventricular system morphometry in fresh and fixed brains found no difference in the ventricular volumes between the two groups, and formalin-fixed brains had a decrease of $1.2 \%$ in length and $1.5 \%$ in width compared to fresh brain tissue, which is quite negligible. ${ }^{4,8)}$

This study also presents two new measurements ALCC-LFCC (distance of the intersection point between the P5-FM line and the lower surface of the corpus callosum to the lower insertion point of the fornix to the corpus callosum) and BLCC-LFCC (distance of the intersection point between the P7FM line and the lower surface of the corpus callosum to the lower insertion point of the fornix to the corpus callosum) which were not defined in former studies. These distances are important during dissection in the prevention of injury to the fornices during interforniceal approach, in which the body of the fornix is split longitudinally in the midline. The fornix connects various structures involved in memory, so damage may result in memory disturbances, making prevention of injury to the fornices especially important. A retrospective study of 17 patients who underwent surgery for a third ventricle lesion found long-term memory and executive functions were frequently impaired. ${ }^{3)}$

The third ventricle region includes many other important neuroanatomic structures involved in motor executive control, and endocrine and vegetative regulations. The corpus callosum and the anterior commissure, septum pellucidum, the motor cortex, and the pyramidal tract are important structures and should be preserved. Damage to the interthalamic adhesion, thalamus, and hypothalamus should also be avoided.

The surgical corridor of the transcallosal-interforniceal approach is bounded by lines perpendicular to the P5 and P7 points and involves the anterior third of the corpus callosum. ${ }^{11)}$ The genu of the corpus callosum, commissure of the fornix, and the motor strip are preserved in surgical approaches using this corridor. Transcallosal approaches from above require careful attention to the pericallosal artery and its branches, from which occasional fine arteries branch out to the falx cerebri. The superior cerebral veins, bridging veins to the superior and inferior sagittal sinuses, and two internal cerebral veins and the choroidal branches also lie in the midline and require special attention during the operative approach. A thorough understanding of the microanatomy of the neural and vascular structures surrounding the third ventricle is essential to improve the surgical results. Preoperative magnetic resonance angiography and cerebral angiography are necessary to assess the topography of the individual patient's vessels. The best operative approach can be determined based on the following: accurate angiography and computed tomography, and magnetic resonance imaging with coronal and sagittal reconstruction to evaluate the lesion and the ventricular size. The incision should be minimized in the cerebral cortex and corpus callosum, and excision 'en bloc' with early coagulation of feeding arteries should be avoided. ${ }^{2}$

Treatment of 11 patients using the direct transcallosal-interforniceal approach showed that this surgical technique is a safe, feasible alternative for the management of a wide spectrum of pathological lesions within this region. The transcallosal-interforniceal approach offers excellent visualization of the entire third ventricle without dependence on hydrocephalus or an extensive extra-axial mass to enhance the exposure, and may be accomplished with the minimum of physiological sequelae using proper planning and technique. ${ }^{1)}$

Another series of 216 operations showed that the transcallosal approach with access to the tumor through the interventricular foramen or between the columns of the fornix was the optimum for radical removal of the tumor. ${ }^{5}$ ) The accurately determined topography of the tumor and the extent of spread beyond the third ventricle guide the choice of the approach.5) In a patient with a small tumor at the level of the foramina, the interventricular foramen will be enlarged and a transforaminal approach should be selected. In a patient with a small tumor at the interventricular foramen or a large tumor, the interforniceal approach should be chosen. ${ }^{11)}$ The transcallosal-transforaminal approach was used via the same surgical corridor in 30 patients with anterior third ventricle tumors, and found that the anatomical landmarks provided adequate guidance to the surgical path and gave excellent results. ${ }^{10)}$ The landmarks P5-ACIS and P7-ACIS are important for the preservation of the cingulate gyrus and 
neighboring limbic system structures. There is a great variation in the depth of the interhemispheric fissure, so the P5-ASCC and P7-ASCC measurements are important. The ALCC-UFCC and BLCCUFCC distances are important to avoid damage to the commissure of the fornix, and the CA-FM distance for avoiding damage to the anterior commissure. ${ }^{10)}$

Careful analysis of magnetic resonance images is mandatory before any surgical procedure for individual surgical planning. This analysis should consider the relationships of the tumor, and also the grade of hydrocephalus and the main anatomical landmarks along the surgical approach. ${ }^{9)}$ Magnetic resonance imaging also provides individualization of surgical interventions in the median structures of brain.

Any microsurgical approach to the third ventricle will attempt to preserve the neurophysiological function and anatomy, with minimal interruption of neuronal pathways and important neuroanatomic structures. Normative morphometric data of the structures involved in the surgical procedure is essential for planning and performance of transcallosal-interforniceal surgical approaches. This study presents detailed morphometric analysis of a large series of cadaver specimens, which showed high variability between individuals in many of the parameters, and highlights the risk of damaging the structures involved in the region during the transcallosal-interforniceal approach.

\section{References}

1) Apuzzo ML, Chikovani OK, Gott PS, Teng EL, Zee CS, Giannotta SL, Weiss MH: Transcallosal, interfornicial approaches for lesions affecting the third ventricle: Surgical considerations and consequences. Neurosurgery 10: 547-554, 1982

2) Gabriel I: Meningioma of the anterior part of the 3rd ventricle. Clinical case. Neurochirurgie 41: 120-123, 1995

3) Godefroy O: Functional anatomy of the third ventricle. Neuropsychological data. Neurochirurgie 46: 175-187, 2000

4) Knudsen PA: Ventriklernes storrelsesforhold i anatomisk normale hjerner fra voksne mennesker. Copenhagen, Denmark, 1958 (Thesis), in Lang J, Stefanec P, Breitenbach W: [Form and measurements of the third ventricle, visual pathway sections and oculomotor nerve]. Neurochirurgia (Stuttg) 26: 1-5, 1983 (Ger, with Eng abstract)

5) Konovalov AN, Gorelyshev SK: [Surgical approach to tumors of the anterior parts of the $3 \mathrm{rd}$ ventricle]. $\mathrm{Zh}$ Vopr Neirokhir Im N N Burdenko 2: 6-12, 1988 (Rus, with Eng abstract)

6) Konovalov AN, Gorelyshev SK: Surgical treatment of anterior third ventricle tumours. Acta Neurochir (Wien) 118: 33-39, 1992

7) Lang J: Surgical anatomy of the hypothalamus. Acta Neurochir (Wien) 75: 5-22, 1985

8) Last RJ, Tompsett JDH: Casts of the cerebral ventricles. Br J Surg 40: 525-543, 1953

9) Lejeune JP, Toussaint P: Surgical anatomy and surgical approaches of the third ventricle. Neurochirurgie 46: 188-202, 2000

10) Winkler PA, Ilmberger J, Krishnan KG, Reulen HJ: Transcallosal interforniceal-transforaminal approach for removing lesions occupying the third ventricular space: clinical and neuropsychological results. Neurosurgery 46: 879-890, 2000

11) Winkler PA, Weis $S$, Buttner A, Raabe A, Amiridze $\mathrm{N}$, Reulen $\mathrm{HJ}$ : The transcallosal interforniceal approach to the third ventricle: anatomic and microsurgical aspects. Neurosurgery 40: 973-982, 1997

12) Winkler PA, Weis S, Wenger E, Herzog C, Dahl A, Reulen HJ: Transcallosal approach to the third ventricle: Normative morphometric data based on magnetic resonance imaging scans, with special reference to the fornix and forniceal insertion. Neurosurgery 45: 309-319, 1999

Address reprint requests to: G. Kayalioglu, M.D., Department of Anatomy, Ege University Faculty of Medicine, TR-35100, Bornova, Izmir, Turkey. e-mail: kayali@med.ege.edu.tr

\section{Commentary}

This is a valuable article that clearly demonstrates that the risk of the transcallosal-interforniceal approach can be prevented by morphometry of the anterior third ventricle region. This study examined the measurements and the major topographic elements of the midline corridor that require consideration. Remarkably, the authors encountered high variability in the measurements in relation to the previous studies and the article makes less clear the variation of these measurements with the most common pathology of the third ventricle region. The review of available literature provides no consistent evidence of normative morphometric measurements. The third ventricle region may be approached by a number of surgical maneuvers that involve different grades of fornix manipulation. This study with 81 adult cadaver hemispheres show two new measurements (ALCC-CA and BLCC-CA) which make the morphometrics more safe, even though great variation of the distances was observed with other studies. The most commonly encountered post-operative problem in the interforniceal approach is transient recent memory loss, with 
resolution of this symptom generally observed within a 21 day period, even though many reports show different destruction of the fornix with absence of detectable amnesia. Another consideration is the development of hemiparesis, related to the midline entry in relation to the cortical venous anatomy and midline retraction. This article is essential to minimize the incidence of complications in the interforniceal approach. In our experience, the use of the transcallosal transchoroidal approach follows a natural route that carries minimum risk of damaging the fornix. The choroidal fissure is certainly one of these natural routes; through it the body of the lateral ventricle communicates with the third ventricle. However, unilateral damage of the fornix produces no deficit. The interforniceal approach carries the potential risk for bilateral damage to the fornix.

Feres CHADDAD, M.D. and Evandro de Oliveira, M.D. Instituto de Ciências Neurológicas São Paulo, Brazil

The authors have to be congratulated for a useful neuromicroanatomical study. There is no doubt that the lesion of one or even both fornices is a difficult functional lesion, which may lead to unsolvable problems. Deteriorated consciousness, combined with a variety of hypothalamic syndromes due to tumorous lesion, and in addition, due to surgery, may represent "insurmountable problems" for the intensivists. The hypothalamic syndromes are difficult to treat, but in cases of preserved consciousness of the patient, the task is much easier. Personally, I have always avoided transcortical approaches, and the same holds for the transcallosal-interforniceal approach. In most of our cases of third ventricular tumors, the interhemispheric-transcallosal, transforaminal approach was used. In cases of eccentric hypothalamic tumors, the interhemispheric-transcallosal-transforaminal or anterior-transventricular direct approach to the tumor was used. ${ }^{1)}$ In cases of optic gliomas and craniopharyngiomas bulging into the third ventricle, the combined approach - pterional and, if necessary, interhemispheric-transcallosaltransforaminal approach-was used. There is no doubt that the normal anatomy is important, but the pathological anatomy in each individual case has to be studied to the full extent preoperatively. During surgery, the most important consideration is not to damage the veins, the fornix, and the perforators. In this respect, coagulation should almost be omitted in these surgeries in order to secure the proper function postoperatively.

\section{Reference}

1) Dolenc VV: Hypothalamic gliomas, in: Advances and Technical Standards in Neurosurgery, vol 25. Wien, New York, Springer, 1999

Vinko V. DOLENC, M.D. Department of Neurosurgery University Medical Center Ljubljana, Slovenia

The cadaveric studies presented in this article offer useful landmarks and measurements when applying the transcallosal-interforniceal approach to the third ventricle, because there are few studies concerning the morphometry of this region. These data are almost consistent with the prior report by Winkler et al. (ref. 11 of this article). Additionally, the authors stress the high variability between individuals in many of the parameters. Such data will no doubt let neurosurgeons know that, because of the high individual variability, measurements should be obtained based on the MRI of each patient and the optimal trajectory to the lesion should be planned individually.

The authors also stress new measurements, the distances of the intersection points between the P5/P7FM line and the upper and lower insertion point of the fornix to the corpus callosum. These distances are important during dissection of the interforniceal raphe posteriorly to avoid damage to the hippocampal commissure, where a number of efferent fibers of the crus of the fornix decussate to the opposite side under the splenium of the corpus callosum. However, I prefer the transforaminal approach with or without the subchoroidal approach rather than the interforniceal approach to gain access into the anterior or middle third ventricle. With the interforniceal approach, the risks of damaging the fornices and the resultant memory disturbance are higher with manipulation or excessive retraction of the fornices.

Ryoji IsHII, M.D.

Department of Neurosurgery

Kawasaki Medical School Kurashiki, Okayama, Japan 